\title{
Asymptotic estimates for $n$-width of fuzzy numbers
}

\author{
Yong J. Han ${ }^{1 *} \mathbb{D}$, Liu Liang ${ }^{2}$ and Guang G. Chen ${ }^{3}$
}

"Correspondence:

hanyj@mail.xhu.edu.cn

'School of Science, Xihua University, Chengdu, P.R. China

Full list of author information is available at the end of the article

\begin{abstract}
$n$-widths in approximation theory characterize how well one can approximate a subset by some "good" subsets of a normed linear space. Especially, $n$-widths of sets of $\mathbb{R}^{N}$ have been studied deeply. Now the following problem is posed: we know that $\mathbb{R}^{N}$ can be embedded in the fuzzy number space $E^{N}$. Is it then possible to define $n$-widths of set $A$ in $E^{N}$ and obtain asymptotic estimates for these $n$-widths?

In this paper, we shall introduce four $n$-widths of $A$ in $E^{N}$ and determine these $n$-widths of Zadeh's extension of diagonal matrices.
\end{abstract}

Keywords: Fuzzy numbers; Approximation theory; n-widths; Zadeh's extension; Diagonal matrix

\section{Introduction}

Let $A$ and $B$ be two subsets of a normed linear space $X$. One may ask: how well $A$ can be approximated by $B$ ? In the theory of $n$-widths of $A$ in $X, B$ will be a simple subspace of $X$. We will consider the possibility of allowing the simple subspaces to vary within $X$ and find the one best adjusted to $A$. In many cases very simple sets may approximate $A$ in an asymptotically optimal manner. It is then possible to judge whether it is worthwhile or not to spend additional time and money in using better but more complicated subspaces. The results of $n$-widths of $A$ in $\mathbb{R}^{N}$ may be found in [1-8].

It is well known that $\mathbb{R}^{N}$ can be embedded in $E^{N}$. Thus if we restrict $d_{s}^{p}$-metric (see Sect. 4) convergence and level convergence on $\mathbb{R}^{N}$, then these types of convergence both become $l_{p}$-metric (induced by $\|\cdot\|_{p}$ ) convergence. Motivated by the study of $n$-widths of $A$ in $\mathbb{R}^{N}$, we introduce definitions of four $n$-widths of $A$ in $E^{N}$. Moreover, asymptotic estimates of these $n$-widths of Zadeh's extension of diagonal matrices are obtained.

\section{Preliminaries}

\subsection{Fuzzy numbers}

For a fuzzy set $u: \mathbb{R}^{N} \rightarrow[0,1]$, suppose that:

(1) $u$ is normal, i.e., there exists $x \in \mathbb{R}^{N}$ such that $u(x)=1$;

(2) $u$ is upper semi-continuous;

(3) $\operatorname{supp} u=\operatorname{cl}\left\{x \in \mathbb{R}^{N}: u(x)>0\right\}$ is compact;

(4) $u$ is fuzzy convex, i.e.,

$$
u(\lambda x+(1-\lambda) y) \geq \min \{u(x), u(y)\}, \quad 0 \leq \lambda \leq 1
$$

(c) The Author(s) 2019. This article is distributed under the terms of the Creative Commons Attribution 4.0 International License (http://creativecommons.org/licenses/by/4.0/), which permits unrestricted use, distribution, and reproduction in any medium, provided you give appropriate credit to the original author(s) and the source, provide a link to the Creative Commons license, and indicate if changes were made. 
for all $x, y \in \mathbb{R}^{N}$. Then $u$ is called a fuzzy number. Let $E^{N}$ be the family of all fuzzy numbers. $\mathbb{R}^{N}$ can be embedded in $\mathbb{E}^{N}$, as any $u \in \mathbb{R}^{N}$ can be viewed as the fuzzy number

$$
\hat{u}(x)= \begin{cases}1, & u=x \\ 0, & u \neq x\end{cases}
$$

For $u, v \in E^{n}, \alpha \in[0,1]$, the $\alpha$-cut of $u$ is defined as follows:

$$
[u]^{\alpha}= \begin{cases}\left\{x \in \mathbb{R}^{N}: u(x) \geq \alpha\right\}, & \text { if } 0<\alpha \leq 1 \\ \operatorname{supp} u, & \text { if } \alpha=0 ;\end{cases}
$$

and the algebraic operations on $E^{N}$ are defined as

$$
[u+v]^{\alpha}=[u]^{\alpha}+[v]^{\alpha}, \quad[k u]^{\alpha}=k[u]^{\alpha}, \quad k \in \mathbb{R}, \alpha \in[0,1] .
$$

If $f: \mathbb{R}^{N} \rightarrow \mathbb{R}^{N}$ is a function, we define Zadeh's extension of $f$ by

$$
\begin{aligned}
& \tilde{f}: E^{N} \rightarrow E^{N}, \\
& \tilde{f}(u)(x)= \begin{cases}\sup _{z \in f^{-1}(x)} u(z), & \text { if } f^{-1}(x) \neq \emptyset ; \\
0, & \text { if } f^{-1}(x)=\emptyset\end{cases}
\end{aligned}
$$

Lemma 1 ([9]) Iff $: \mathbb{R}^{N} \rightarrow \mathbb{R}^{N}$ is continuous, then $\tilde{f}$ is a well-defined function and

$$
[\widetilde{f}(u)]^{\alpha}=f\left([u]^{\alpha}\right), \quad \forall \alpha \in[0,1], \forall u \in E^{N} .
$$

\section{$2.2 n$-Widths of diagonal matrix}

Definition 1 ([10]) Let $(X,\|\cdot\|)$ be a normed linear space, and $A \subseteq X$.

(1) The Kolmogorov $n$-width of $A$ in $X$ is defined by

$$
d_{n}(A ; X)=\inf _{X_{n}} \sup _{x \in A} \inf _{y \in X_{n}}\|x-y\|,
$$

where the left-most infimum is taken over all $n$-dimensional subspace $X_{n}$ of $X$.

(2) The Bernstein $n$-width of $A$ in $X$ is defined by

$$
\begin{aligned}
b_{n}(A ; X) & =\sup _{X_{n+1}} \sup \left\{\lambda: \lambda S\left(X_{n+1}\right) \subseteq A\right\} \\
& =\sup _{X_{n+1}} \inf _{x \in \partial\left(A \cap X_{n+1}\right)}\|x\|,
\end{aligned}
$$

where $X_{n+1}$ is any $(n+1)$-dimensional subspace of $X$, and $S\left(X_{n+1}\right)$ is the unit ball of $X_{n+1}$.

(3) The Gelfand $n$-width of $A$ in $X$ is defined as

$$
d^{n}(A ; X)=\inf _{L^{n}} \sup _{x \in A \cap L^{n}}\|x\|,
$$

where the infimum is taken over all subspaces $L^{n}$ of $X$ of codimension $n$. 
(4) The linear $n$-width is given by

$$
\delta_{n}(A ; X)=\inf _{P_{n}(A)} \sup _{x \in A}\left\|x-P_{n}(x)\right\|
$$

where the infimum is taken over all continuous linear operators $P_{n}$ of $X$ into $X$ of rank $n$.

Lemma 2 ([11]) Let $X_{n+1}$ be any $(n+1)$-dimensional subspace of a normed linear space $(X,\|\cdot\|)$, and let $S\left(X_{n+1}\right)$ denote the unit ball of $X_{n+1}$. Then

$$
d_{k}\left(S\left(X_{n+1}\right) ; X\right)=1, \quad k=1,2, \ldots, n .
$$

Let $l_{p}^{N}$ be the $N$-dimensional normed spaces of $x=\left(x_{1}, \ldots, x_{N}\right) \in \mathbb{R}^{N}$, with the normed

$$
\|x\|_{p}= \begin{cases}\left(\sum_{i=1}^{N}\left|x_{i}\right|^{p}\right)^{1 / p}, & 1 \leq p<\infty \\ \max _{1 \leq i \leq N}\left|x_{i}\right|, & p=\infty\end{cases}
$$

Let $D=\operatorname{diag}\left\{D_{1}, \ldots, D_{N}\right\}$ be an $N \times N$ diagonal matrix. Without loss of generality, we assume that

$$
D_{1} \geq D_{2} \geq \cdots \geq D_{N}>0
$$

$n$-widths of $\mathfrak{D}_{p}=\left\{D x:\|x\|_{p} \leq 1\right\}$ can be found in $[1,2,10]$.

Theorem A $([2,10])$ For $1 \leq p \leq \infty$,

$$
d_{n}\left(\mathfrak{D}_{p} ; l_{p}^{N}\right)=d^{n}\left(\mathfrak{D}_{p} ; l_{p}^{N}\right)=b_{n}\left(\mathfrak{D}_{p} ; l_{p}^{N}\right)=\delta_{n}\left(\mathfrak{D}_{p} ; l_{p}^{N}\right)=D_{n+1}
$$

Theorem B ([1]) Given $1 \leq q \leq p \leq \infty$. Let $1 / r=1 / q-1 / p$. Then

$$
d_{n}\left(\mathfrak{D}_{p} ; l_{q}^{N}\right)=d^{n}\left(\mathfrak{D}_{p} ; l_{q}^{N}\right)=b_{n}\left(\mathfrak{D}_{p} ; l_{q}^{N}\right)=\delta_{n}\left(\mathfrak{D}_{p} ; l_{q}^{N}\right)=\left(\sum_{k=n+1}^{N} D_{k}^{r}\right)^{1 / r}
$$

\section{3 -Widths of fuzzy numbers}

The following notation will be used throughout this paper. Let $X_{n}$ be an $n$-dimensional subspace of $\mathbb{R}^{N}, L^{n}$ be subspaces of $\mathbb{R}^{N}$ of codimension $n . S\left(X_{n}\right)$ denotes the unit ball of $X_{n}$. Set

$$
\begin{aligned}
& \widetilde{X_{n}}=\left\{u: u \in E^{N},[u]^{0} \subseteq X_{n}\right\}, \\
& \widetilde{L^{n}}=\left\{u: u \in E^{N},[u]^{0} \subseteq L^{n}\right\}, \\
& S\left(\widetilde{X_{n+1}}\right)=\left\{u: d(u, \hat{0}) \leq 1, u \in \widetilde{X_{n+1}}\right\} .
\end{aligned}
$$

Let $\widetilde{P_{n}}$ be Zadeh's extension of the continuous linear operators $P_{n}$ of $\mathbb{R}^{N}$ into $\mathbb{R}^{N}$ of rank $n$.

Definition 2 Let $\left(E^{N}, d\right)$ be a metric space, and $A \subseteq E^{N}$. 
(1) The Kolmogorov $n$-width of $A$ in $E^{N}$ is defined by

$$
d_{n}\left(A ; E^{N}\right)=\inf _{\widetilde{X}_{n}} \sup _{u \in A} \inf _{v \in \widetilde{X}_{n}} d(u, v)
$$

where the left-most infimum is taken over all $\widetilde{X_{n}} \subseteq \mathbb{E}^{N}$.

(2) The Bernstein $n$-width of $A$ in $E^{N}$ is defined by

$$
b_{n}\left(A ; E^{N}\right)=\sup _{X_{n+1}} \sup \left\{\lambda: \lambda \geq 0, \lambda S\left(\widetilde{X_{n+1}}\right) \subseteq A\right\} .
$$

(3) The Gelfand $n$-width of $A$ in $E^{N}$ is defined as

$$
d^{n}\left(A ; E^{N}\right)=\inf _{\widetilde{L^{n}}} \sup _{u \in A \cap \widetilde{L^{n}}} d(u, \hat{0})
$$

where the infimum is taken over all subspaces $\widetilde{L^{n}}$ of $E^{N}$.

(4) The linear $n$-width of $A$ in $E^{N}$ is given by

$$
\delta_{n}\left(A ; E^{N}\right)=\inf _{\widetilde{P_{n}}} \sup _{u \in A} d\left(u, \widetilde{P_{n}}(u)\right)
$$

where the infimum is taken over all $\widetilde{P_{n}}$.

Proposition 1 Let $\left(E^{N}, d\right)$ be a metric space, and $A \subseteq E^{N}$.

(i) $\delta_{n}\left(A ; E^{N}\right) \geq d_{n}\left(A ; E^{N}\right)$.

(ii) $\delta_{n}\left(A ; E^{N}\right) \geq d^{n}\left(A ; E^{N}\right)$.

Proof Let $\widetilde{P_{n}}$ be Zadeh's extension of the continuous linear operators $P_{n}$ of $\mathbb{R}^{N}$ into $\mathbb{R}^{N}$ of rank $n$.

(i) From Lemma 1 and $\operatorname{rank} P_{n}=n$, we know that there exists an $n$-dimensional subspace $X_{n}$ of $\mathbb{R}^{N}$ subject to the following relation:

$$
\left[\widetilde{P_{n}}(u)\right]^{0}=P_{n}\left([u]^{0}\right) \subseteq X_{n}, \quad u \in A \subseteq E^{N}
$$

Then $\widetilde{P_{n}}(u) \in \widetilde{X_{n}}$, i.e., $\widetilde{P_{n}}(A) \subseteq \widetilde{X_{n}}$. By the definitions of $d_{n}\left(A ; E^{N}\right)$ and $\delta_{n}\left(A ; E^{N}\right)$

$$
\delta_{n}\left(A ; E^{N}\right) \geq d_{n}\left(A ; E^{N}\right) .
$$

(ii) If $\widetilde{P_{n}}(u)=\hat{0}$, then

$$
\left[\widetilde{P_{n}}(u)\right]^{0}=P_{n}\left([u]^{0}\right)=\{0\}
$$

and $[u]^{0} \subseteq L^{n}$, i.e., $u \in \widetilde{L^{n}}$. Therefore

$$
\sup _{u \in A} d\left(u, \widetilde{P_{n}}(u)\right) \geq \sup _{\substack{u \in A \\ \widetilde{P}_{n}(u)=0}} d(u, \hat{0}),
$$

whence it follows that $\delta_{n}\left(A ; E^{N}\right) \geq d^{n}\left(A ; E^{N}\right)$. 


\section{$4 n$-Widths of $\tilde{D}$}

We first choose a suitable metric $d(\cdot, \cdot)$ on $E^{N}$ to establish a relation between $\|x-y\|_{p}$ and $d(u, v), x, y \in l_{p}^{N}, u, v \subset E^{N}$. Then the distance between subsets of $E^{N}$ can be estimated by $\|x-y\|_{p}$.

Let $\mathcal{K}\left(\mathbb{R}^{N}\right)$ be the family of nonempty compact subsets of $l_{p}^{N}$. If $A, B \in \mathcal{K}\left(\mathbb{R}^{N}\right), 1 \leq p<\infty$, the Hausdorff distance between $A$ and $B$ is defined by

$$
d_{H}^{p}(A, B)=\max \left\{\sup _{a \in A} \inf _{b \in B}\|a-b\|_{p}, \sup _{b \in B} \inf _{a \in A}\|a-b\|_{p}\right\} .
$$

For $u, v \in E^{N}, 1 \leq s<\infty, \alpha \in[0,1]$, we define

$$
d_{s}^{p}(u, v)=\left(\int_{0}^{1} d_{H}^{p}\left([u]^{\alpha},[v]^{\alpha}\right)^{s} d \alpha\right)^{1 / s},
$$

then $d_{s}^{p}$ is called the $L_{s}$-metric on $E^{N}[12]$. Let $L_{s, p}^{N}:=\left(E^{N}, d_{s}^{p}\right)$.

Proposition $2([12])\left(E^{N}, d_{s}^{p}\right)$ is a metric space for $1 \leq s, p<\infty$.

Let $D=\operatorname{diag}\left\{D_{1}, \ldots, D_{N}\right\}, D_{1} \geq D_{2} \geq \cdots \geq D_{N}>0$, be an $N \times N$ diagonal matrix, and $\widetilde{D}$ be Zadeh's extension of $D$.

Lemma 3 Let $u \in E^{N}, k \in \mathbb{R}, 1 \leq s<\infty, \alpha \in[0,1]$. Then

$$
d_{s}^{p}(\widetilde{D} u, k \hat{0})=\left(\int_{0}^{1}\left(\sup _{a \in[u]^{\alpha}}\|D a\|_{p}\right)^{s} d \alpha\right)^{1 / s}
$$

and

$$
d_{s}^{p}(\widetilde{D}(k u), \hat{0})=|k| d_{s}^{p}(\widetilde{D}(u), \hat{0}) .
$$

Proof Since supp $\hat{0}=\{0\}$, it follows that

$$
\begin{aligned}
d_{H}^{p}\left([\widetilde{D} u]^{\alpha}, k[\hat{0}]^{\alpha}\right) & =d_{H}^{p}\left(D\left([u]^{\alpha}\right), k[\hat{0}]^{\alpha}\right) \\
& =\max \left\{\sup _{a \in[u]^{\alpha}} \inf _{b \in k[\hat{0}]^{\alpha}}\|D a-b\|_{p}, \sup _{b \in k[\hat{0}]^{\alpha}} \inf _{a \in[u]^{]^{\prime}}}\|D a-b\|_{p}\right\} \\
& =\sup _{a \in[u]^{\alpha}}\|D a\|_{p} .
\end{aligned}
$$

Hence

$$
d_{s}^{p}(\widetilde{D} u, k \hat{0})=\left(\int_{0}^{1}\left(\sup _{a \in[u]^{\alpha}}\|D a\|_{p}\right)^{s} d \alpha\right)^{1 / s} .
$$

Similarly, we can get the second equation.

In this paper we are concerned with the estimate of $n$-widths of

$$
\widetilde{\mathfrak{D}_{s, p}}=\left\{\widetilde{D} u: u \in E^{N}, d_{s}^{p}(u, \hat{0}) \leq 1\right\}
$$


and

$$
\widetilde{\mathfrak{D}_{s, p}^{-1}}=\left\{\widetilde{D^{-1}} u: u \in E^{N}, d_{s}^{p}(u, \hat{0}) \leq 1\right\}
$$

It is often the case, see examples in [10], that a very simple form of $b_{n}(A ; X)=$ $\sup _{X_{n+1}} \inf _{x \in \partial\left(A \cap X_{n+1}\right)}\|x\|$ is used. We introduce a similar definition of $b_{n}\left(\widetilde{\mathfrak{D}_{s, p}} ; L_{s, p}^{N}\right)$ for easy computation.

Definition 3 For $1 \leq s, p<\infty$,

$$
b_{n}\left(\widetilde{\mathfrak{D}_{s, p}} ; L_{s, p}^{N}\right)=\sup _{\widetilde{X_{n+1}}} \inf _{\substack{u \in \bar{X}_{n+1} \\ d_{s}^{p}(u, \hat{0})=1}} d(\widetilde{D} u, \hat{0})
$$

Now we state our main results.

Theorem 1 For $1 \leq s, p<\infty$,

$$
d_{n}\left(\widetilde{\mathfrak{D}_{s, p}} ; L_{s, p}^{N}\right)=d^{n}\left(\widetilde{\mathfrak{D}_{s, p}} ; L_{s, p}^{N}\right)=b_{n}\left(\widetilde{\mathfrak{D}_{s, p}} ; L_{s, p}^{N}\right)=\delta_{n}\left(\widetilde{\mathfrak{D}_{s, p}} ; L_{s, p}^{N}\right)=D_{n+1}
$$

Theorem 2 Given $1 \leq s<\infty, 1 \leq q \leq p<\infty$. Let $1 / r=1 / q-1 / p$. Then

$$
d_{n}\left(\widetilde{\mathfrak{D}_{s, p}} ; L_{s, q}^{N}\right)=d^{n}\left(\widetilde{\mathfrak{D}_{s, p}} ; L_{s, q}^{N}\right)=\delta_{n}\left(\widetilde{\mathfrak{D}_{s, p}} ; L_{s, q}^{N}\right)=\left(\sum_{k=n+1}^{N} D_{k}^{r}\right)^{1 / r}
$$

Remark For $1 \leq q \leq p<\infty$, Theorem 1 and 2 are obvious generalizations of Theorems $\mathrm{A}$ and $B$.

Before proving these two theorems, we need some lemmas.

Lemma 4 For $1 \leq s, p<\infty$,

(i) $\delta_{n}\left(\widetilde{\mathfrak{D}_{s, p}} ; L_{s, p}^{N}\right) \geq d_{n}\left(\widetilde{\mathfrak{D}_{s, p}} ; L_{s, p}^{N}\right) \geq b_{n}\left(\widetilde{\mathfrak{D}_{s, p}} ; L_{s, p}^{N}\right)$.

(ii) $\delta_{n}\left(\widetilde{\mathfrak{D}_{s, p}} ; L_{s, p}^{N}\right) \geq d^{n}\left(\widetilde{\mathfrak{D}_{s, p}} ; L_{s, p}^{N}\right) \geq b_{n}\left(\widetilde{\mathfrak{D}_{s, p}} ; L_{s, p}^{N}\right)$.

Proof From Proposition 1 the following results are known:

$$
\delta_{n}\left(\widetilde{\mathfrak{D}_{s, p}} ; L_{s, p}^{N}\right) \geq d_{n}\left(\widetilde{\mathfrak{D}_{s, p}} ; L_{s, p}^{N}\right), \quad \delta_{n}\left(\widetilde{\mathfrak{D}_{s, p}} ; L_{s, p}^{N}\right) \geq d^{n}\left(\widetilde{\mathfrak{D}_{s, p}} ; L_{s, p}^{N}\right)
$$

Now we prove that $d_{n}\left(\widetilde{\mathfrak{D}_{s, p}} ; L_{s, p}^{N}\right) \geq b_{n}\left(\widetilde{\mathfrak{D}_{s, p}} ; L_{s, p}^{N}\right)$. If $\lambda S\left(\widetilde{X_{n+1}}\right) \subseteq \widetilde{\mathfrak{D}_{s, p}}$, then from the definition of $d_{n}\left(A ; E^{N}\right)$

$$
d_{n}\left(\widetilde{\mathfrak{D}_{s, p}} ; L_{s, p}^{N}\right) \geq d_{n}\left(\lambda S\left(\widetilde{X_{n+1}}\right) ; L_{s, p}^{N}\right) \geq d_{n}\left(\lambda S\left(X_{n+1}\right) ; L_{s, p}^{N}\right)
$$

For $\hat{a} \in \lambda S\left(X_{n+1}\right), v \in \widetilde{X_{n}}$, a direct computation shows that

$$
d_{H}^{p}\left(\{a\},[v]^{\alpha}\right)=\sup _{b \in[v]^{\alpha}}\|a-b\|_{p} \geq \inf _{b \in X_{n}}\|a-b\|_{p}
$$


and

$$
d_{s}^{p}(\hat{a}, v)=\left(\int_{0}^{1} d_{H}^{p}\left(\{a\},[v]^{\alpha}\right)^{s} d \alpha\right)^{1 / s} \geq \inf _{b \in X_{n}}\|a-b\|_{p}
$$

Therefore

$$
\sup _{\hat{a} \in \lambda S\left(X_{n+1}\right)} \inf _{v \in X_{n+1}} d_{s}^{p}(\hat{a}, v) \geq \sup _{a \in \lambda S\left(X_{n+1}\right)} \inf _{b \in X_{n}}\|a-b\|_{p},
$$

which implies that

$$
d_{n}\left(\lambda S\left(X_{n+1}\right) ; L_{s, p}^{N}\right) \geq d_{n}\left(\lambda S\left(X_{n+1}\right) ; l_{p}^{N}\right) .
$$

From (1) and Lemma 2

$$
d_{n}\left(\widetilde{\mathfrak{D}_{s, p}} ; L_{s, p}^{N}\right) \geq \lambda
$$

By the definition of $b_{n}\left(A ; E^{N}\right)$ in Definition $2(2)$, we have $d_{n}\left(\widetilde{\mathfrak{D}_{s, p}} ; L_{s, p}^{N}\right) \geq b_{n}\left(\widetilde{\mathfrak{D}_{s, p}} ; L_{s, p}^{N}\right)$.

The proof of $d^{n}\left(\widetilde{\mathfrak{D}_{s, p}} ; L_{s, p}^{N}\right) \geq b_{n}\left(\widetilde{\mathfrak{D}_{s, p}} ; L_{s, p}^{N}\right)$ is totally analogous to the proof of $d_{n}\left(\widetilde{\mathfrak{D}_{s, p}}\right.$; $\left.L_{s, p}^{N}\right) \geq b_{n}\left(\widetilde{\mathfrak{D}_{s, p}} ; L_{s, p}^{N}\right)$, here we omit it. The theorem is proved.

Let $a=\left(a_{1}, \ldots, a_{N}\right) \in \mathbb{R}^{N}, A, B \subseteq \mathbb{R}^{N}$, we write $a \perp A$ if $\sum_{i=1}^{N} a_{i} x_{i}=0$ for all $x=\left(x_{1}\right.$, $\left.\ldots, x_{N}\right) \in A$ and $A \perp B$ if $\sum_{i=1}^{N} a_{i} b_{i}=0$ for $\forall a=\left(a_{1}, \ldots, a_{N}\right) \in A, b=\left(b_{1}, \ldots, b_{N}\right) \in B$. Set

$$
A^{\perp}=\left\{y \in \mathbb{R}^{N}: \sum_{i=1}^{N} x_{i} y_{i}=0, \forall x=\left(x_{1}, \ldots, x_{N}\right) \in A\right\} .
$$

Let $u \in E^{N}, C \subseteq E^{N}$, we write $u \perp C$ if $[u]^{0} \perp[v]^{0}, \forall v \in C$.

Lemma 5 For $u \in E^{N}$, the following properties are equivalent.

(i) $u \perp \widetilde{X_{N-n}}$.

(ii) $u \in \widetilde{X_{n}}$.

Proof (i) $\Rightarrow$ (ii). Since $u \perp \widetilde{X_{N-n}}$, we know that for $\forall v \in \widetilde{X_{N-n}}$, i.e., $[v]^{0} \subseteq X_{N-n}$, we have $[u]^{0} \perp[v]^{0}$.

$$
[u]^{0} \subset X_{N-n}^{\perp} .
$$

Note that $X_{N-n}^{\perp}=X_{n}$. Then (ii) follows.

(ii) $\Rightarrow$ (i). If $u \in \widetilde{X}_{n}$, i.e., $[u]^{0} \subseteq X_{n}$, then for all $x \in[u]^{0}$ there is an $(N-n)$-dimensional subspace $X_{N-n}$ such that $x \perp X_{N-n}$. Consequently, we have $[u]^{0} \perp X_{N-n}$, i.e., $u \perp \widetilde{X_{N-n}}$.

Lemma 6 For $1 \leq s, p<\infty, n<N$,

$$
b_{n}\left(\widetilde{\mathfrak{D}_{s, p}}, L_{s, q}^{N}\right) d^{N-n-1}\left(\widetilde{\mathfrak{D}_{s, q}^{-1}}, L_{s, p}^{N}\right)=1
$$


Proof By the definition of $d^{N-n-1}\left(\widetilde{\mathfrak{D}_{s, q}^{-1}}, L_{s, p}^{N}\right)$ and Lemma 5, we have

$$
\begin{aligned}
d^{N-n-1}\left(\widetilde{\mathfrak{D}_{s, p}^{-1}}, L_{s, q}^{N}\right) & =\frac{\min }{\max _{X_{N-n-1}}} d_{\substack{u \in X_{n+1} \\
d_{s}^{p}(u, \hat{0}) \leq 1}}\left(\widetilde{D^{-1}} u, \hat{0}\right) \\
& =\underset{X_{N-n-1}}{\min } \underset{\substack{u \perp X_{N-n-1} \\
u \neq \hat{0}}}{\max } \frac{d_{s}^{q}\left(\widetilde{D^{-1}} u, \hat{0}\right)}{d_{s}^{p}(u, \hat{0})} \\
& =\underset{X_{N-n-1}}{\min } \underset{\substack{u \perp X_{N-n-1} \\
u \neq \hat{0}}}{\max }\left[\frac{d_{s}^{p}(u, \hat{0})}{d_{s}^{q}\left(\widetilde{D^{-1}} u, \hat{0}\right)}\right]^{-1} .
\end{aligned}
$$

Setting $v=\widetilde{D^{-1}} u$, by Lemma 1 we have

$$
[v]^{\alpha}=D^{-1}[u]^{\alpha}, \quad \forall \alpha \in[0,1]
$$

and

$$
[u]^{\alpha}=D D^{-1}[u]^{\alpha}=D\left[\widetilde{D^{-1}} u\right]^{\alpha}=D[v]^{\alpha}=[\widetilde{D} v]^{\alpha}, \quad \forall \alpha \in[0,1] .
$$

Since $D$ is invertible, hence $v \perp \widetilde{X_{N-n-1}}$. Now

$$
\begin{aligned}
d^{N-n-1}\left(\widetilde{\mathfrak{D}_{s, q}^{-1}}, L_{s, p}^{N}\right) & =\underset{\min _{N-n-1}}{\max }\left[\widetilde{\substack{v \perp X_{N-n-1} \\
v \neq \hat{0}}}\left[\frac{d_{s, p}(\widetilde{D} v, \hat{0})}{d_{s}^{q}(v, \hat{0})}\right]^{-1}\right. \\
& =\left[\max _{\widetilde{X_{n+1}}} \min _{\substack{v \in \widetilde{X_{n+1}} \\
v \neq \hat{0}}} \frac{d_{s}^{p}(\widetilde{D} v, \hat{0})}{d_{s}^{q}(v, \hat{0})}\right]^{-1} \\
& =\left[b_{n}\left(\widetilde{\mathfrak{D}_{s, p}}, L_{s, q}^{N}\right)\right]^{-1} .
\end{aligned}
$$

Proof of Theorem 1 Let $P_{n}=\operatorname{diag}\left(D_{1}, \ldots, D_{n}, 0, \ldots, 0\right)$. For any $u \in E^{N}$,

$$
\begin{aligned}
\left.d_{H}^{p}\left([\widetilde{D} u]^{\alpha}\right),\left[\widetilde{P}_{n} u\right]^{\alpha}\right) & =d_{H}^{p}\left(D\left([u]^{\alpha}\right), P_{n}\left([u]^{\alpha}\right)\right) \\
& =\max \left\{\sup _{a \in[u]^{\alpha}} \inf _{b \in[u]^{\alpha}}\left\|D a-P_{n} b\right\|_{p}, \sup _{b \in[u]^{\alpha}} \inf _{a \in[u]^{\alpha}}\left\|D a-P_{n} b\right\|_{p}\right\} \\
& =\max _{a \in[u]^{\alpha}}\left\|\left(D-P_{n}\right) a\right\|_{p} .
\end{aligned}
$$

Then

$$
\begin{aligned}
\delta_{n}\left(\widetilde{\mathfrak{D}_{s, p}} ; L_{s, p}^{N}\right) & \leq \max _{d_{s}^{p}(u, \hat{0}) \leq 1} d_{s}^{p}\left(\widetilde{D} u, \widetilde{P_{n}} u\right) \\
& =\max _{u \neq \hat{0}} \frac{d_{s}^{p}\left(\widetilde{D} u, \widetilde{P_{n}} u\right)}{d_{s}^{p}(u, \hat{0})} \\
& =\max _{u \neq \hat{0}} \frac{\left(\int_{0}^{1}\left(\max _{a \in[u]^{\alpha}}\left\|\left(D-P_{n}\right) a\right\|_{p}\right)^{s} d \alpha\right)^{1 / s}}{\left(\int_{0}^{1}\left(\max _{a \in[u]^{\alpha}}\|a\|_{p}\right)^{s} d \alpha\right)^{1 / s}} \\
& =\max _{u \neq \hat{0}} \frac{\left(\int_{0}^{1}\left(\max _{a \in[u]^{\alpha}}\left(\sum_{i=n+1}^{N}\left|D_{i} a_{i}\right|^{p}\right)^{1 / p}\right)^{s} d \alpha\right)^{1 / s}}{\left(\int_{0}^{1}\left(\max _{a \in[u]^{\alpha}}\|a\|_{p}\right)^{s} d \alpha\right)^{1 / s}}
\end{aligned}
$$




$$
\begin{aligned}
& \leq \max _{u \neq \hat{0}} \frac{D_{n+1}\left(\int_{0}^{1}\left(\max _{a \in[u]^{\alpha}}\left(\sum_{i=n+1}^{N}\left|a_{i}\right|^{p}\right)^{1 / p}\right)^{s} d \alpha\right)^{1 / s}}{\left(\int_{0}^{1}\left(\max _{a \in[u]^{\alpha}}\|a\|_{p}\right)^{s} d \alpha\right)^{1 / s}} \\
& \leq D_{n+1} .
\end{aligned}
$$

In a similar way,

$$
\delta_{n}\left(\widetilde{\mathfrak{D}_{s, p}^{-1}} ; L_{s, p}^{N}\right) \leq 1 / D_{n+1}
$$

From Lemma 6

$$
\begin{aligned}
b_{n}\left(\widetilde{\mathfrak{D}_{s, p}} ; L_{s, p}^{N}\right) & =\left(d^{M-n-1}\left(\widetilde{\mathfrak{D}_{s, p}^{-1}} ; L_{s, p}^{N}\right)\right)^{-1} \\
& \geq\left(\delta_{n}\left(\widetilde{\mathfrak{D}_{s, p}^{-1}} ; L_{s, p}^{N}\right)\right)^{-1} .
\end{aligned}
$$

Thus

$$
b_{n}\left(\widetilde{\mathfrak{D}_{s, p}} ; L_{s, p}^{N}\right) \geq D_{n+1}
$$

By Lemma 4 , we prove that these four $n$-widths equal $D_{n+1}$.

Let $1 \leq p, q<\infty$, and $1 / p+1 / p^{\prime}=1 / q+1 / q^{\prime}=1$. We use the notation $x^{\prime}(x)=\left\langle x, x^{\prime}\right\rangle$ for $x \in l_{p}^{N}, x^{\prime} \in l_{p^{\prime}}^{N}$, and similarly for $l_{q}^{N}$. Diagonal matrix $D: l_{p}^{N} \rightarrow l_{q}^{N}$ has an adjoint $D^{\prime}: l_{q^{\prime}}^{N} \rightarrow$ $l_{p^{\prime}}^{N}$, defined by $\left\langle D x, y^{\prime}\right\rangle=\left\langle x, D^{\prime} y^{\prime}\right\rangle$ for $x \in l_{p}^{N}$ and $y^{\prime} \in l_{q^{\prime}}^{N}$. It is well known that $D=D^{\prime}$. Let $L^{n}$ be subspaces of $l_{p}^{N}$ of codimension $n$ and $\widetilde{L^{n}}=\left\{u \in E^{N}:[u]^{0} \subseteq L^{n}\right\}$, set

$$
\begin{aligned}
& L_{\perp}^{n}=\left\{x^{\prime}: x^{\prime} \in l_{p^{\prime}}^{N},\left\langle x, x^{\prime}\right\rangle=0, \text { all } x \in L^{n}\right\}, \\
& \widetilde{L_{\perp}^{n}}=\left\{v: v \in E^{N},[v]^{0} \subset L_{\perp}^{n}\right\} .
\end{aligned}
$$

It is well known that $\operatorname{dim} L_{\perp}^{n}=n$.

Lemma 7 Let $1 \leq s, p, q<\infty$, and $1 / p+1 / p^{\prime}=1 / q+1 / q^{\prime}=1$. Then

$$
d_{n}\left(\widetilde{\mathfrak{D}_{s, p}} ; L_{s, q}^{N}\right) \geq d^{n}\left(\widetilde{\mathfrak{D}_{s, q^{\prime}}} ; L_{s, p^{\prime}}^{N}\right) .
$$

Proof Let $\widetilde{A_{p}}=\left\{u \in E^{N}: d_{s}^{p}(u, \hat{0}) \leq 1\right\}$. Then

$$
\begin{aligned}
\sup _{u \in \widetilde{A_{p}} \cap \widetilde{L^{n}}} \sup _{a \in[u]^{0}}\|D a\|_{q} & =\sup _{u \in \widetilde{A_{p}} \cap \widetilde{L^{n}}} \sup _{a \in[u]^{0}} \sup _{\left\|b^{\prime}\right\|_{q^{\prime}} \leq 1}\left\langle D a, b^{\prime}\right\rangle \\
& \leq \sup _{u \in \widetilde{A_{p}} \cap \widetilde{L^{n}}} \sup _{a \in[u]^{0}} \sup _{v \in \widetilde{A_{q^{\prime}}}} \sup _{b^{\prime} \in[v]^{\alpha}}\left\langle D a, b^{\prime}\right\rangle \\
& =\sup _{v \in \widetilde{A_{q^{\prime}}}} \sup _{u \in \widetilde{A_{p}} \cap \widetilde{L}^{n}} \sup _{b^{\prime} \in[v]^{\alpha}} \sup _{a \in[u]^{0}}\left\langle a, D^{\prime} b^{\prime}\right\rangle .
\end{aligned}
$$

Let $u \in \widetilde{A_{p}} \cap \widetilde{L^{n}}$ and $a^{\prime} \in L_{\perp}^{n}$. Then $\left\langle a, a^{\prime}\right\rangle=0, \forall a \in[u]^{0}$. Hence

$$
\left\langle a, D^{\prime} b^{\prime}\right\rangle=\left\langle a, D^{\prime} b^{\prime}-a^{\prime}\right\rangle \leq\|a\|_{p}\left\|D^{\prime} b^{\prime}-a^{\prime}\right\|_{p^{\prime}} \leq\left\|D^{\prime} b^{\prime}-a^{\prime}\right\|_{p^{\prime}} .
$$


For $u^{\prime} \in \widetilde{L_{\perp}^{n}}$, we have

$$
\sup _{b^{\prime} \in[v]^{\alpha}} \sup _{a \in[u]^{0}}\left\langle a, D^{\prime} b^{\prime}\right\rangle \leq \sup _{b^{\prime} \in[v]^{\alpha}} \inf _{a^{\prime} \in\left[u^{\prime}\right]^{\alpha}}\left\|D b^{\prime}-a^{\prime}\right\|_{p^{\prime}}
$$

Following (3), (4), and $D=D^{\prime}$

$$
\begin{aligned}
\sup _{v \in \widetilde{A_{q^{\prime}}}} \sup _{u \in \widetilde{A_{p}} \cap \widetilde{L^{n}}} \sup _{b^{\prime} \in[v]^{\alpha}} \sup _{a \in[u]^{0}}\left\langle a, D^{\prime} b^{\prime}\right\rangle & \leq \sup _{v \in \widetilde{A_{q^{\prime}}}} \inf _{u^{\prime} \in \widetilde{L_{\perp}^{n}}} \sup _{b^{\prime} \in[v]^{\alpha}} \inf _{a^{\prime} \in\left[u^{\prime}\right]^{\alpha}}\left\|D b^{\prime}-a^{\prime}\right\|_{p^{\prime}} \\
& \leq \sup _{v \in \widetilde{A_{q^{\prime}}}} \inf _{u^{\prime} \in \widetilde{L_{\perp}^{n}}} d_{s}^{p^{\prime}}\left(u^{\prime}, \widetilde{D} v\right) .
\end{aligned}
$$

Combining Lemma 3, (3), and (5)

$$
\sup _{u \in \widetilde{A_{p}} \cap \widetilde{L^{n}}} d_{s}^{q}(\widetilde{D} u, \hat{0}) \leq \sup _{v \in \widetilde{A_{q^{\prime}}}} \inf _{u^{\prime} \in \widetilde{L}_{\perp}^{\underline{n}}} d_{s}^{p^{\prime}}\left(u^{\prime}, \widetilde{D} v\right)
$$

then taking the infimum over $\widetilde{L^{n}}$, we have the result.

Proof of Theorem 2 We first prove that $\delta_{n}\left(\widetilde{\mathfrak{D}_{s, p}} ; L_{s, q}^{N}\right) \leq\left(\sum_{k=n+1}^{N} D_{k}^{r}\right)^{1 / r}$.

Let $P_{n}=\operatorname{diag}\left(D_{1}, \ldots, D_{n}, 0, \ldots, 0\right)$. For any $u \in E^{N}$, as (2) in the proof of Theorem 1

$$
\begin{aligned}
\left.d_{H}^{q}\left([\widetilde{D} u]^{\alpha}\right),\left[\widetilde{P}_{n} u\right]^{\alpha}\right) & =\sup _{a \in[u]^{\alpha}}\left\|\left(D-P_{n}\right) a\right\|_{q} \\
& =\sup _{a \in[u]^{\alpha}}\left(\sum_{k=n+1}^{N}\left|D_{k} a_{k}\right|^{q}\right)^{1 / q} .
\end{aligned}
$$

From $1 / r=1 / q-1 / p$ and Hölder's inequality

$$
\left(\sum_{k=n+1}^{N}\left|D_{k} a_{k}\right|^{q}\right)^{1 / q} \leq\left(\sum_{k=n+1}^{N}\left|D_{k}\right|^{r}\right)^{1 / r}\left(\sum_{k=n+1}^{N}\left|a_{k}\right|^{p}\right)^{1 / p}
$$

we have

$$
\begin{aligned}
\left.d_{H}^{q}\left([\widetilde{D} u]^{\alpha}\right),\left[\widetilde{P}_{n} u\right]^{\alpha}\right) & \leq \sup _{a \in[u]^{\alpha}}\left(\sum_{k=n+1}^{N}\left|D_{k}\right|^{r}\right)^{1 / r}\left(\sum_{k=n+1}^{N}\left|a_{k}\right|^{p}\right)^{1 / p} \\
& \leq\left(\sum_{k=n+1}^{N}\left|D_{k}\right|^{r}\right)^{1 / r} \sup _{a \in[u]^{\alpha}}\left(\sum_{k=1}^{N}\left|a_{k}\right|^{p}\right)^{1 / p} \\
& =\left(\sum_{k=n+1}^{N}\left|D_{k}\right|^{r}\right)^{1 / r} d_{H}^{p}\left([u]^{\alpha},[\hat{0}]^{\alpha}\right) .
\end{aligned}
$$

Then

$$
\begin{aligned}
\delta_{n}\left(\widetilde{\mathfrak{D}_{s, p}} ; L_{s, q}^{N}\right) & \leq \max _{d_{s}^{p}(u, \hat{0}) \leq 1} d_{s}^{q}\left([\widetilde{D} u]^{\alpha}\right),\left[\widetilde{\left.\left.P_{n} u\right]^{\alpha}\right)}\right. \\
& \leq \max _{d_{s}^{p}(u, \hat{0}) \leq 1}\left(\int_{0}^{1} d_{H}^{q}\left(D\left([u]^{\alpha}\right), P_{n}\left([u]^{\alpha}\right)\right)^{s} d \alpha\right)^{1 / s}
\end{aligned}
$$




$$
\begin{aligned}
& \leq \max _{d_{s}^{p}(u, \hat{0}) \leq 1}\left(\sum_{k=n+1}^{N}\left|D_{k}\right|^{r}\right)^{1 / r} d_{s}^{p}(u, \hat{0}) \\
& \leq\left(\sum_{k=n+1}^{N}\left|D_{k}\right|^{r}\right)^{1 / r} .
\end{aligned}
$$

Now we are to prove $d^{n}\left(\widetilde{\mathfrak{D}_{s, p}} ; L_{s, q}^{N}\right) \geq\left(\sum_{k=n+1}^{N}\left|D_{k}\right|^{r}\right)^{1 / r}$. From Lemma 3

$$
\begin{aligned}
\left.d_{H}^{q}\left([\widetilde{D} u]^{\alpha}\right),[\hat{0}]^{\alpha}\right) & =d_{H}^{q}\left(D\left([u]^{\alpha}\right),[\hat{0}]^{\alpha}\right) \\
& =\max _{a \in[u]^{\alpha}}\|D a\|_{q} \\
& =\max _{a \in[u]^{\alpha}}\left(\sum_{k=1}^{N}\left|D_{k} a_{k}\right|^{q}\right)^{1 / q}
\end{aligned}
$$

and

$$
\left.d_{H}^{p}\left([u]^{\alpha}\right),[\hat{0}]^{\alpha}\right)=\max _{a \in[u]^{\alpha}}\left(\sum_{k=1}^{N}\left|a_{k}\right|^{p}\right)^{1 / p} .
$$

By the definition of $d^{n}\left(\widetilde{\mathfrak{D}_{s, p}}, L_{s, q}^{N}\right)$ and Theorem B

$$
d^{n}\left(\widetilde{\mathfrak{D}_{s, p}}, L_{s, q}^{N}\right) \geq d^{n}\left(\mathfrak{D}_{p}, L_{s, q}^{N}\right)=d^{n}\left(\mathfrak{D}_{p}, l_{q}^{N}\right)=\left(\sum_{k=n+1}^{N} D_{k}^{r}\right)^{1 / r} .
$$

Combine (6), (7), and Proposition 1(ii),

$$
\delta_{n}\left(\widetilde{\mathfrak{D}_{s, p}} ; L_{s, q}^{N}\right)=d^{n}\left(\widetilde{\mathfrak{D}_{s, p}}, L_{s, q}^{N}\right)=\left(\sum_{k=n+1}^{N} D_{k}^{r}\right)^{1 / r} .
$$

Similarly, we can have

$$
\delta_{n}\left(\widetilde{\mathfrak{D}_{s, q^{\prime}}} ; L_{s, p^{\prime}}^{N}\right)=\left(\sum_{k=n+1}^{N} D_{k}^{r}\right)^{1 / r}
$$

and

$$
d^{n}\left(\widetilde{\mathfrak{D}_{s, q^{\prime}}}, L_{s, p^{\prime}}^{N}\right) \geq\left(\sum_{k=n+1}^{N} D_{k}^{r}\right)^{1 / r} .
$$

By Proposition 1(i) and Lemma 7

$$
d_{n}\left(\widetilde{\mathfrak{D}_{s, p}} ; L_{s, q}^{N}\right)=\left(\sum_{k=n+1}^{N} D_{k}^{r}\right)^{1 / r}
$$

The theorem is proved. 
Acknowledgements

Not applicable.

\section{Funding}

This work was supported by the Key Scientific Research Fund of Xihua University (Grant No. 21412621) and the National Natural Science Foundation of China (Grant No. 15233593).

\section{Availability of data and materials}

Not applicable.

\section{Competing interests}

The authors declare that they have no competing interests.

\section{Authors' contributions}

All authors contributed equally to the manuscript. All authors read and approved the final manuscript.

\section{Author details}

'School of Science, Xihua University, Chengdu, P.R. China. ${ }^{2}$ Institute of Applied Mathematics, School of Science, Xihua University, Chengdu, P.R. China. ${ }^{3}$ Department of Postgraduate, Xihua University, Chengdu, P.R. China.

\section{Publisher's Note}

Springer Nature remains neutral with regard to jurisdictional claims in published maps and institutional affiliations.

Received: 20 July 2018 Accepted: 21 March 2019 Published online: 29 March 2019

\section{References}

1. Pietsch, A.: s-Numbers of operators in Banach spaces. Stud. Math. 51, 201-223 (1974)

2. Mityagin, B.S., Tichomirov, V.M.: Asymptotic characteristic of compact sets in linear spaces. In: Proc. Fourth All-Union Soviet Math. Congress, Vol. II. Sectional Lecture, vol. 299. Nauka, Leningrad (1964)

3. Sofman, L.B.: Diameters of octahedra. Mat. Zametki 5, 429-436 (1969)

4. Tichomirov, V.M., Babadjanov, S.B.: On the width of a functional class in the space $\mathbf{L}_{p}(p \geq 1)$. Izv. Akad. Nauk UzSSR Ser. Fiz.-Mat. Nauk 2, 24-30 (1967)

5. Hutton, C.V., Morrell, J.S., Retherford, J.R.: Diagonal operators, approximation numbers, and Kolmogoroff diameters. J. Approx. Theory 16, 48-80 (1976)

6. Pinkus, A.: Matrices and $n$-widths. Linear Algebra Appl. 27, 245-278 (1979)

7. Melkman, A.A.: The distance of a subspace of $\mathbb{R}^{m}$ from its axes and $n$-widths of octahedra. J. Approx. Theory 42 , 245-256 (1984)

8. Kashin, B.S.: Diameters of some finite-dimensional sets and classes of smooth functions. Izv. Akad. Nauk SSSR 41, 334-351 (1977)

9. Nguyen, H.T: A note on the extension principle for fuzzy sets. J. Math. Anal. Appl. 64, 369-380 (1978)

10. Pinkus, A.: $n$-Widths in Approximation Theory. Springer, Berlin (1985)

11. Gohberg, I.C., Krein, M.G.: Introduction to the Theory of Linear Nonselfadjoint Operators in Hilbert Space. Transl. Math. Monographs. Am. Math. Soc., Providence (1969)

12. Diamond, P., Kloeden, P.E.: Metric Spaces of Fuzzy Sets, Theory and Application. World Scientific, London (1994)

\section{Submit your manuscript to a SpringerOpen ${ }^{\circ}$ journal and benefit from:}

- Convenient online submission

- Rigorous peer review

- Open access: articles freely available online

- High visibility within the field

- Retaining the copyright to your article

Submit your next manuscript at $\boldsymbol{~ s p r i n g e r o p e n . c o m ~}$ 